\title{
Double Elzaki Transform Decomposition Method for Solving Third Order Korteweg-De-Vries Equations
}

\author{
Moh A. Hassan1, Tarig M. Elzaki² \\ ${ }^{1}$ Mathematics Department, Faculty of Mathematical \& Computer Science, Gezira University, Wad Madani, Sudan \\ ${ }^{2}$ Mathematics Department, Faculty of Sciences and Arts-Alkamil, University of Jeddah, Jeddah, Saudi Arabia \\ Email: moh.hassan512@yahoo.com,Tarig.alzaki@gmail.com
}

How to cite this paper: Hassan, M.A. and Elzaki, T.M. (2021) Double Elzaki Transform Decomposition Method for Solving Third Order Korteweg-De-Vries Equations. Journal of Applied Mathematics and Physics, 9, 21-30.

https://doi.org/10.4236/jamp.2021.91003

Received: November 3, 2020

Accepted: January 3, 2021

Published: January 6, 2021

Copyright $\odot 2021$ by author(s) and Scientific Research Publishing Inc. This work is licensed under the Creative Commons Attribution International License (CC BY 4.0).

http://creativecommons.org/licenses/by/4.0/

\begin{abstract}
In this study, we used Double Elzaki Transform (DET) coupled with Adomian polynomial to produce a new method to solve Third Order KortewegDe Vries Equations (KdV) equations. We will provide the necessary explanation for this method with addition some examples to demonstrate the effectiveness of this method.
\end{abstract}

\section{Keywords}

Double Elzaki Transform, Adomian Polynomial, Korteweg-De Vries Equations

\section{Introduction}

In 1985, Two Dutchmen, D. J. Korteweg and G. de Vrie derived a nonlinear partial differential equation, well known by the Korteweg-de Vries (KdV) equation, to model the height of the surface of shallow water in the presence of solitary wave's. The KdV equation also describes the propagation of plasma waves in a dispersive medium.

Third order Korteweg-de-Vries (KdV) equation of the form

$$
u_{t}+a u u_{x}+b u_{x x x}=0
$$

with the initial conditions:

$$
u(x, 0)=f(x)
$$

where $a$ and $b$ are constants.

So many methods and approaches have been made to find the approximate analytic solutions and numerical solutions of KdV equations, such as Adomian 
Decomposition Method (ADM) [1], Variation Iteration Method (VIM) [1], Homotopy Perturbation Method (HPM) [1], Homotopy Perturbation Method using Elzaki Transform [2], Homotopy Perturbation Method using Laplace Transform [3], Adomian Decomposition Method using Elzaki Transform [4], Numerical solutions to a linear $\mathrm{KdV}$ equation on unbounded domain [5], The numerical solutions of $\mathrm{KdV}$ equation using radial basis functions [6], Numerical solution of separated solitary waves for $\mathrm{KdV}$ equation through finite element technique [7].

In this paper, we study a new method to solutions of $\mathrm{KdV}$ equations namely Double Elzaki Transform Decomposition Method (DETDM), this method is its capability of combining easy integral transform Double ELzaki Transform (DET) [8] and an effective method for solving non-linear partial differential equations, namely Adomian Decomposition Method [1].

Several examples are given as follows to illustrate this method to explain its effectiveness.

\section{Basic Definitions of Double Elzaki Transform}

Definition: Let $f(x, t), t, x \in R^{+}$be a function which can be expressed as a convergent infinite series, then its Double Elzaki Transform given by:

$$
E_{2}[f(x, t), u, v]=T(u, v)=u v \int_{0}^{\infty} \int_{0}^{\infty} f(x, t) \mathrm{e}^{-\left(\frac{x}{u}+\frac{t}{v}\right)} \mathrm{d} x \mathrm{~d} t, x, t>0 .
$$

where $u, v$ are complex values.

To obtain double Elzaki transform of partial derivatives we use integration by parts [8], and then we have:

$$
\begin{gathered}
E_{2}\left[\frac{\partial f}{\partial x}\right]=\frac{1}{u} T(u, v)-u T(0, v) \\
E_{2}\left[\frac{\partial^{2} f}{\partial x^{2}}\right]=\frac{1}{u^{2}} T(u, v)-T(0, v)-u \frac{\partial}{\partial x} T(0, v) \\
E_{2}\left[\frac{\partial f}{\partial t}\right]=\frac{1}{v} T(u, v)-v T(u, 0) \\
E_{2}\left[\frac{\partial^{2} f}{\partial t^{2}}\right]=\frac{1}{v^{2}} T(u, v)-T(u, 0)-v \frac{\partial}{\partial t} T(u, 0) \\
E_{2}\left[\frac{\partial^{2} f}{\partial x \partial t}\right]=\frac{1}{u v} T(u, v)-\frac{v}{u} T(u, 0)-\frac{u}{v} T(0, v)+u v T(0,0)
\end{gathered}
$$

Proof:

$$
E_{2}\left[\frac{\partial f}{\partial x}\right]=u v \int_{0}^{\infty} \int_{0}^{\infty} \frac{\partial}{\partial x} f(x, t) \mathrm{e}^{-\left(\frac{x}{u}+\frac{t}{v}\right)} \mathrm{d} x \mathrm{~d} t=v \int_{0}^{\infty} \mathrm{e}^{-\frac{t}{v}}\left[u \int_{0}^{\infty} \mathrm{e}^{-\frac{x}{u}} \frac{\partial}{\partial x} f(x, t) \mathrm{d} x\right] \mathrm{d} t
$$

The inner integral gives $\frac{1}{u} T(u, t)-u f(0, t)$ 


$$
\begin{gathered}
\Rightarrow E_{2}\left[\frac{\partial f}{\partial x}\right]=\frac{v}{u} \int_{0}^{\infty} \mathrm{e}^{-\frac{t}{v}} T(u, t) \mathrm{d} t-u v \int_{0}^{\infty} \mathrm{e}^{-\frac{t}{v}} f(0, t) \mathrm{d} t \\
\Rightarrow E_{2}\left[\frac{\partial f}{\partial x}\right]=\frac{1}{u} T(u, v)-u T(0, v)
\end{gathered}
$$

Also $E_{2}\left[\frac{\partial f}{\partial t}\right]=\frac{1}{v} T(u, v)-v T(u, 0)$

$$
\begin{aligned}
E_{2}\left[\frac{\partial^{2} f(x, t)}{\partial x^{2}}\right] & =u v \int_{0}^{\infty} \int_{0}^{\infty} \frac{\partial^{2} f(x, t)}{\partial x^{2}} \mathrm{e}^{-\left(\frac{x}{u}+\frac{t}{v}\right)} \mathrm{d} x \mathrm{~d} t \\
& =v \int_{0}^{\infty} \mathrm{e}^{-\frac{t}{v}}\left[u \int_{0}^{\infty} \frac{\partial^{2} f(x, t)}{\partial x^{2}} \mathrm{e}^{-\frac{x}{u}} \mathrm{~d} x\right] \mathrm{d} t
\end{aligned}
$$

The inner integral: $u \int_{0}^{\infty} \frac{\partial^{2} f(x, t)}{\partial x^{2}} \mathrm{e}^{-\frac{x}{u}} \mathrm{~d} x=\frac{T(u, t)}{u^{2}}-f(0, t)-u \frac{\partial f(0, t)}{\partial x}$.

By taking Elzaki transform with respect to $t$ for above integral we get:

$$
E_{2}\left[\frac{\partial^{2} f(x, t)}{\partial x^{2}}\right]=\frac{1}{u^{2}} T(u, v)-T(0, v)-u \frac{\partial}{\partial x} T(0, v)
$$

Similarly:

$$
E_{2}\left[\frac{\partial^{2} f(x, t)}{\partial t^{2}}\right]=\frac{1}{v^{2}} T(u, v)-T(u, 0)-v \frac{\partial}{\partial t} T(u, 0)
$$

\section{Theorems of Convergence of Double Elzaki Transform:}

Theorem 3.1. Let the function $f(x, t)$ is continuous in the $x t$ - plane, if the integral converges at $u=u_{0}, v=v_{0}$ then the integral,

$u v \int_{0}^{\infty} \int_{0}^{\infty} f(x, t) \mathrm{e}^{-\left(\frac{x}{u}+\frac{t}{v}\right)} \mathrm{d} x \mathrm{~d} t$ is convergence for $u<u_{0}, v<v_{0}$.

For the proof we will use the following theorems.

Theorem 3.2. Suppose that: $v \int_{0}^{\infty} f(x, t) \mathrm{e}^{-\frac{t}{v}} \mathrm{~d} t$, converges at $v=v_{0}$, then the integral converges for $v<v_{0}$

Proof

$$
\text { Let } \alpha(x, t)=v_{0} \int_{0}^{t} f(x, s) \mathrm{e}^{-\frac{s}{v_{0}}} \mathrm{~d} s, 0<t<\infty
$$

Clearly $\alpha(x, 0)=0$ and $\lim _{t \rightarrow \infty} \alpha(x, t)$ exist.

By fundamental theorem of calculus we have:

$$
\alpha_{t}(x, t)=v_{0} f(x, t) \mathrm{e}^{-\frac{t}{v_{0}}}
$$

If we choose $\epsilon_{1}$ and $R_{1}$ such that $\left(0<\epsilon_{1}<R_{1}\right)$ and using Equation (6) we get:

$$
v \int_{\epsilon_{1}}^{R_{1}} f(x, t) \mathrm{e}^{-\frac{t}{v}} \mathrm{~d} t=v \int_{\epsilon_{1}}^{R_{1}} \frac{1}{v_{0}} \mathrm{e}^{\frac{t}{v_{0}}} \alpha_{t}(x, t) \mathrm{e}^{-\frac{t}{v}} \mathrm{~d} t=\frac{v}{v_{0}} \int_{\epsilon_{1}}^{R_{1}} \alpha_{t}(x, t) \mathrm{e}^{-\left(\frac{v_{0}-v}{v v_{0}}\right) t} \mathrm{~d} t
$$


Integrating the last integral by parts to gives:

$$
\begin{aligned}
& \frac{v}{v_{0}} \int_{\epsilon_{1}}^{R_{1}} \alpha_{t}(x, t) \mathrm{e}^{-\left(\frac{v_{0}-v}{v v_{0}}\right) t} \mathrm{~d} t \\
& =\frac{v}{v_{0}}\left[\left(\alpha(x, t) \mathrm{e}^{-\left(\frac{v_{0}-v}{v v_{0}}\right) t}\right)_{\epsilon_{1}}^{R_{1}}-\int_{\epsilon_{1}}^{R_{1}} \alpha(x, t) \cdot \mathrm{e}^{-\left(\frac{v_{0}-v}{v v_{0}}\right) t} \cdot\left(-\left(\frac{v_{0}-v}{v v_{0}}\right)\right) \mathrm{d} t\right] \\
& =\frac{v}{v_{0}}\left[\alpha\left(x, R_{1}\right) \mathrm{e}^{-\left(\frac{v_{0}-v}{v v_{0}}\right) R_{1}}-\alpha\left(x, \epsilon_{1}\right) \mathrm{e}^{-\left(\frac{v_{0}-v}{v v_{0}}\right) \epsilon_{1}}+\left(\frac{v_{0}-v}{v v_{0}}\right) \int_{\epsilon_{1}}^{R_{1}} \alpha(x, t) \mathrm{e}^{-\left(\frac{v_{0}-v}{v v_{0}}\right) t} \mathrm{~d} t\right]
\end{aligned}
$$

Now let $\epsilon_{1} \rightarrow 0, R_{1} \rightarrow \infty$, if $v<v_{0}$, then we have

$$
v \int_{0}^{\infty} f(x, t) \mathrm{e}^{-\frac{t}{v}} \mathrm{~d} t=\left(\frac{v_{0}-v}{v_{0}^{2}}\right) \int_{0}^{\infty} \alpha(x, t) \mathrm{e}^{-\left(\frac{v_{0}-v}{v v_{0}}\right) t} \mathrm{~d} t
$$

Now if the integral on the right converges then the theorem is proved.

By using limit test for convergence we get:

$$
\lim _{t \rightarrow \infty} t^{2} \alpha(x, t) \mathrm{e}^{-\left(\frac{v_{0}-v}{v v_{0}}\right) t}=\lim _{t \rightarrow \infty}\left(\frac{t^{2}}{\mathrm{e}^{\left(\frac{v_{0}-v}{v v_{0}}\right) t}}\right) \cdot \lim _{t \rightarrow \infty}(\alpha(x, t))
$$

The first limit equal zero at $t \rightarrow \infty$ if $v<v_{0}$ and the second limit exist, then

$$
\lim _{t \rightarrow \infty} t^{2} \alpha(x, t) \mathrm{e}^{-\left(\frac{v_{0}-v}{v v_{0}}\right) t}=0 \text {, finite. }
$$

Then the integral $v \int_{0}^{\infty} f(x, t) \mathrm{e}^{-\frac{t}{v}} \mathrm{~d} t$ is converges at $v<v_{0}$.

Theorem 3.3. Suppose that: $u \int_{0}^{\infty} f(x, t) \mathrm{e}^{-\frac{x}{u}} \mathrm{~d} x$, converges at $u=u_{0}$, then the integral converges for $u<u_{0}$

\section{Proof}

Prove, of this theorem is same as the method in theorem (3.2).

Now the proof of the theorem (3.1) is as follows

$$
u v \int_{0}^{\infty} \int_{0}^{\infty} f(x, t) \mathrm{e}^{-\left(\frac{x}{u}+\frac{t}{v}\right)} \mathrm{d} x \mathrm{~d} t=u \int_{0}^{\infty} \mathrm{e}^{-\frac{x}{u}}\left[v \int_{0}^{\infty} \mathrm{e}^{-\frac{t}{v}} f(x, t) \mathrm{d} t\right] \mathrm{d} x
$$

By using theorem (3.2) and theorem (3.3) we see the integral in RHS of Equation (10) is converges for $u<u_{0}, v<v_{0}$, hence the integral

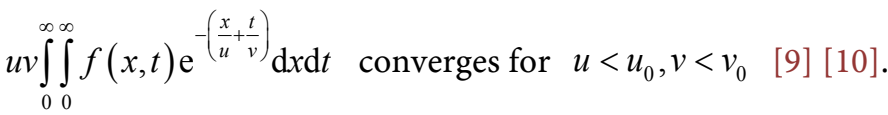

\section{Double Elzaki Transform Decomposition Method (DETDM)}

The main focus of this study is to solve the Third Order Korteweg-De Vries Equations (KdV) equations. Firstly we show how to use Double Elzaki Transform 
Decomposition Method (DETDM) to solve the general nonlinear partial differential equations [11].

Consider a general partial differential equation with the initial condition of the following form:

$$
\begin{gathered}
L u(x, t)+R u(x, t)+N u(x, t)=g(x, t), \\
u(x, 0)=h(x), \quad u_{t}(x, 0)=f(x) .
\end{gathered}
$$

where, $L$ is the second order linear differential operator $L=\frac{\partial^{2}}{\partial t^{2}}, R$ is the linear differential operator of less order then $L, N$ represents the general nonlinear differential operator and $g(x, t)$ is the source term.

Taking the double Elzaki Transform on both sides of Equation (11) and single Elzaki Transform of Equation (12), we get:

$$
\begin{gathered}
E_{2}(L u(x, t))+E_{2}(R u(x, t))+E_{2}(N u(x, t))=E_{2}(g(x, t)), \\
E(u(x, 0))=E(h(x))=T(u, 0) \text { and } E\left(u_{t}(x, 0)\right)=E(f(x))=\frac{\partial}{\partial t} T(u, 0) .
\end{gathered}
$$

To substitute Equation (14) in (13), after using Equation (4), we get:

$$
\begin{aligned}
E_{2}(u(x, t))= & v^{2} E_{2}(g(x, t))+v^{2} E(h(x))+v^{3} E(f(x)) \\
& -v^{2} E_{2}(R u(x, t))-v^{2} E_{2}(N u(x, t)) .
\end{aligned}
$$

Now, with the application of the inverse Double Elzaki Transform on both side of Equation (15) we get:

$$
u(x, t)=G(x, t)-E_{2}^{-1}\left[v^{2} E_{2}[R u(x, t)+N u(x, t)]\right] .
$$

where $G(x, t)$ represents the terms arising from the source term and the prescribed initial conditions.

After that we represent solution as an infinite series given below,

$$
u(x, t)=\sum_{n=0}^{\infty} u_{n}(x, t),
$$

and the nonlinear term can be written as follow,

$$
N u(x, t)=\sum_{n=0}^{\infty} A_{n}(u),
$$

where, $A_{n}(u)$ are Adomian polynomial and it can be calculated by formula given below:

$$
A_{n}=\frac{1}{n !} \frac{\mathrm{d}^{n}}{\mathrm{~d} \lambda^{n}}\left[N\left(\sum_{i=0}^{\infty} \lambda^{i} u_{i}\right)\right]_{\lambda=0}, n=0,1,2,3, \cdots
$$

To substitute (17) and (18) in (16), we get:

$$
\sum_{n=0}^{\infty} u_{n}(x, t)=G(x, t)-E_{2}^{-1}\left[v^{2} E_{2}\left(R \sum_{n=0}^{\infty} u_{n}(x, t)+\sum_{n=0}^{\infty} A_{n}\right)\right] .
$$

Then from Equation (20) we get: 


$$
\begin{aligned}
& u_{0}(x, t)=G(x, t), \\
& u_{1}(x, t)=-E_{2}^{-1}\left[v^{2} E_{2}\left[R u_{0}(x, t)+A_{0}\right]\right], \\
& u_{2}(x, t)=-E_{2}^{-1}\left[v^{2} E_{2}\left[R u_{1}(x, t)+A_{1}\right]\right] .
\end{aligned}
$$

In general, the recursive relation is given by:

$$
u_{n}(x, t)=-E_{2}^{-1}\left[v^{2} E_{2}\left[R u_{n-1}(x, t)+A_{n-1}\right]\right], n \geq 1 .
$$

Finally, we approximate the solution $u(x, t)$ by the series:

$$
u(x, t)=\lim _{N \rightarrow \infty} \sum_{n=0}^{\infty} u_{n}(x, t) .
$$

\section{Applications}

Now we are demonstrated the effectiveness of this method, by solving the following third Order Korteweg-De Vries Equations (KdV) equations.

Example 1: Consider the following $\mathrm{KdV}$ equations

$$
u_{t}+6 u u_{x}+u_{x x x}=0
$$

with initial condition:

$$
u(x, 0)=x .
$$

Take the double Elzaki transform to both sides of Equation (24), we get:

$$
\frac{T(u, v)}{v}-v T(u, 0)=-E_{2}\left(6 u u_{x}+u_{x x x}\right),
$$

Take single Elzaki transform to initial condition we get:

$$
E(u(x, 0))=T(u, 0)=E(x)=u^{3},
$$

Substitute Equation (27) in Equation (26), we obtain:

$$
T(u, v)=v^{2} u^{3}-v E_{2}\left(6 u u_{x}+u_{x x x}\right) .
$$

Take the inverse double Elzaki transform to both sides of Equation (28), we obtain:

$$
u(x, t)=x-E_{2}^{-1}\left[v E_{2}\left(6 u u_{x}+u_{x x x}\right)\right] .
$$

From the Adomian decomposition method, rewrite Equation (29) as follows,

$$
\sum_{n=0}^{\infty} u_{n}(x, t)=x-E_{2}^{-1}\left[v E_{2}\left(6 \sum_{n=0}^{\infty} A_{n}(u)+\sum_{n=0}^{\infty}\left(u_{n}\right)_{x x x}\right)\right] .
$$

where, $A_{n}(u)$ areAdomian polynomials that represent the nonlinear terms.

The first few components of $A_{n}(u)$ are given by:

$$
\begin{aligned}
& A_{0}(u)=u_{0}\left(u_{0}\right)_{x}, \\
& A_{1}(u)=\left(u_{0}\right)_{x} u_{1}+u_{0}\left(u_{1}\right)_{x}, \\
& A_{2}(u)=\left(u_{0}\right)_{x} u_{2}+\left(u_{1}\right)_{x} u_{1}+\left(u_{2}\right)_{x} u_{0}, \\
& A_{3}(u)=\left(u_{0}\right)_{x} u_{3}+\left(u_{1}\right)_{x} u_{2}+\left(u_{2}\right)_{x} u_{1}+\left(u_{3}\right)_{x} u_{0},
\end{aligned}
$$


By comparing both sides of Equation (22), we get:

$$
\begin{gathered}
u_{0}(x, t)=x, \\
u_{n+1}(x, t)=-E_{2}^{-1}\left[v E_{2}\left[6 A_{n}(u)+\left(u_{n}\right)_{x x x}\right]\right], n \geq 0 .
\end{gathered}
$$

Then:

$$
\begin{aligned}
u_{1}(x, t) & =-E_{2}^{-1}\left[v E_{2}\left[6 A_{0}(u)+\left(u_{0}\right)_{x x x}\right]\right] \\
& =-E_{2}^{-1}\left[v E_{2}(6 x)\right]=-E_{2}^{-1}\left[6 v^{3} u^{3}\right]=-6 x t, \\
u_{2}(x, t)= & -E_{2}^{-1}\left[v E_{2}\left[6 A_{1}(u)+\left(u_{1}\right)_{x x x}\right]\right] \\
= & -E_{2}^{-1}\left[v E_{2}(-72 x t)\right]=E_{2}^{-1}\left[72 v^{4} u^{3}\right]=36 x t^{2},
\end{aligned}
$$

By similar way we get:

$$
u_{3}(x, t)=-216 x t^{3} .
$$

And so on, then the first four terms of the decomposition series for Equation (24) are given by:

$$
u(x, t)=x-6 x t+36 x t^{2}-216 x t^{3}+\cdots,
$$

This can be written as:

$$
u(x, t)=x\left[1-6 t+(6 t)^{2}-(6 t)^{3}+\cdots\right],
$$

The solution in a closed form is given by:

$$
u(x, t)=\frac{x}{1+6 t},|t|<1 .
$$

Example 2: Consider the following $\mathrm{KdV}$ equations

$$
u_{t}-6 u u_{x}+u_{x x x}=0 \text {, }
$$

with initial condition:

$$
u(x, 0)=\frac{1}{6}(x-1) .
$$

Take the double Elzaki transform to both sides of equation (40), we get:

$$
\frac{T(u, v)}{v}-v T(u, 0)=E_{2}\left(6 u u_{x}-u_{x x x}\right),
$$

Take single Elzaki transform to initial condition we get:

$$
E(u(x, 0))=T(u, 0)=E\left(\frac{1}{6}(x-1)\right)=\frac{1}{6}\left(u^{3}-u^{2}\right),
$$

Substitute Equation (42) in Equation (41), we obtain:

$$
T(u, v)=\frac{1}{6}\left(v^{2} u^{3}-v^{2} u^{2}\right)+v E_{2}\left(6 u u_{x}-u_{x x x}\right) .
$$

Take the inverse double Elzaki transform to both sides of Equation (44), we obtain:

$$
u(x, t)=\frac{1}{6}(x-1)+E_{2}^{-1}\left[v E_{2}\left(6 u u_{x}-u_{x x x}\right)\right] .
$$


From the Adomian decomposition method, rewrite Equation (45) as follows,

$$
\sum_{n=0}^{\infty} u_{n}(x, t)=\frac{1}{6}(x-1)+E_{2}^{-1}\left[v E_{2}\left(6 \sum_{n=0}^{\infty} A_{n}(u)-\sum_{n=0}^{\infty}\left(u_{n}\right)_{x x x}\right)\right] \text {. }
$$

where, $A_{n}(u)$ are the Adomian polynomials that represent the nonlinear terms.

The first few components of $A_{n}(u)$ are given by:

$$
\begin{aligned}
& A_{0}(u)=u_{0}\left(u_{0}\right)_{x}, \\
& A_{1}(u)=\left(u_{0}\right)_{x} u_{1}+u_{0}\left(u_{1}\right)_{x}, \\
& A_{2}(u)=\left(u_{0}\right)_{x} u_{2}+\left(u_{1}\right)_{x} u_{1}+\left(u_{2}\right)_{x} u_{0}, \\
& A_{3}(u)=\left(u_{0}\right)_{x} u_{3}+\left(u_{1}\right)_{x} u_{2}+\left(u_{2}\right)_{x} u_{1}+\left(u_{3}\right)_{x} u_{0},
\end{aligned}
$$

By comparing both sides of Equation (46), we get:

$$
\begin{gathered}
u_{0}(x, t)=\frac{1}{6}(x-1), \\
u_{n+1}(x, t)=E_{2}^{-1}\left[v E_{2}\left[6 A_{n}(u)-\left(u_{n}\right)_{x x x}\right]\right], n \geq 0 .
\end{gathered}
$$

Then:

$$
\begin{aligned}
u_{1}(x, t) & =E_{2}^{-1}\left[v E_{2}\left[6 A_{0}(u)-\left(u_{0}\right)_{x x x}\right]\right] \\
& =E_{2}^{-1}\left[v E_{2}\left(6 \cdot \frac{1}{36}(x-1)\right)\right] \\
& =E_{2}^{-1}\left[\frac{1}{6}\left(v^{3} u^{3}-v^{3} u^{2}\right)\right] \\
& =\frac{1}{6}(x-1) t, \\
u_{2}(x, t) & =E_{2}^{-1}\left[v E_{2}\left[6 A_{1}(u)-\left(u_{1}\right)_{x x x}\right]\right] \\
& =E_{2}^{-1}\left[v E_{2}\left(6 \cdot \frac{1}{6}(x-1) \cdot \frac{1}{3} t\right)\right] \\
& =E_{2}^{-1}\left[v E_{2}\left(\frac{1}{3} x t-\frac{1}{3} t\right)\right] \\
& =E_{2}^{-1}\left[\frac{1}{3} u^{3} v^{4}-\frac{1}{3} u^{2} v^{4}\right] \\
& =\frac{1}{6}(x-1) t^{2},
\end{aligned}
$$

By similar way we get:

$$
u_{3}(x, t)=\frac{1}{6}(x-1) t^{3}
$$

And so on. Then the first four terms of the decomposition series for Equation (40), is given by:

$$
u(x, t)=\frac{1}{6}(x-1)\left(1+t+t^{2}+t^{3}+\cdots\right),
$$

The solution in a closed form is given by: 


$$
u(x, t)=\frac{1}{6}\left(\frac{x-1}{1-t}\right),|t|<1 .
$$

\section{Conclusion}

This method is very effective for solving non-linear partial differential equations in general, and as a special case, the Third Order Korteweg-De Vries (KdV) equations. It can be applied to higher order Korteweg-De Vries Equations.

\section{Availability of Data and Materials}

Data sharing not applicable to this article as no datasets were generated or analyzed during the current study.

\section{Authors' Contributions}

The authors read and agreed the final manuscript.

\section{Conflicts of Interest}

The authors declare no conflicts of interest regarding the publication of this paper.

\section{References}

[1] Wazwaz, A.M. (2009) Partial Differential Equations Solitary Waves' Theory. Springer, Berlin. https://doi.org/10.1007/978-3-642-00251-9

[2] Chavan, S.S. and Panchal, M.M. (2014) Solution of Third Order Korteweg-De Vries Equation by Homotopy Perturbation Method Using Elzaki Transform. International Journal for Research in Applied Science and Engineering Technology, 2, 366-369.

[3] Eljaily, M.H. and Tarig, M.E. (2015) Homotopy Perturbation Transform Method for Solving Korteweg-Devries (Kdv) Equation. Pure and Applied Mathematics Journal, 4, 264-268. https://doi.org/10.11648/j.pamj.20150406.17

[4] Ige, O.E., Heilio, M., Oderinu, R.A. and Elzaki, T.M. (2019) Adomian Polynomial and Elzaki Transform Method of Solving Third Order Korteweg-De Vries Equations. Pure and Applied Mathematics, 15, 261-277. https://doi.org/10.12732/ijam.v32i3.7

[5] Zheng, C., Wen, X. and Han, H. (2008) Numerical Solution to a Linearized Kdv Equation on Unbounded Domain. Numerical Methods for Partial Differential Equations, 24, 383-399. https://doi.org/10.1002/num.20267

[6] Dag, I. and Dereli, Y. (2008) Numerical Solutions of Kdv Equation Using Radial Basis Functions. Applied Mathematical Modelling, 32, 535-546. https://doi.org/10.1016/j.apm.2007.02.001

[7] Kapoor, S., Rawat, S. and Dhawan, S. (2012) Numerical Investigation of Separated Solitary Waves Solution for kdv Equation through Finite Element Technique. International Journal of Computer Applications, 40, 27-33. https://doi.org/10.5120/5049-7463

[8] Elzaki, T.M. and Hilal, E.M. (2012) Solution of Telegraph Equation by Modified of Double Sumudu Transform "Elzaki Transform”. Mathematical Theory and Modeling, 2, 95-103. 
[9] Idrees, M.I., Ahmed, Z., Awais, M. and Perveen, Z. (2018) On the Convergence of Double Elzaki Transform. International Journal of Advanced and Applied Sciences, 5, 19-24. https://doi.org/10.21833/ijaas.2018.06.003

[10] Alderremy, A.A. and Elzakib, T.M. (2018) On the New Double Integral Transform for Solving Singular System of Hyperbolic Equations. Journal of Nonlinear Sciences and Applications, 11, 1207-1214. https://doi.org/10.22436/jnsa.011.10.08

[11] Hassan, M.A. and Elzaki, T.M. (2020) Double Elzaki Transform Decomposition Method for Solving Non-Linear Partial Differential Equations. Journal of Applied Mathematics and Physics, 8, 1463-1471. https://doi.org/10.4236/jamp.2020.88112 